\title{
[PHYSICS IN SPACE]
}

\section{THERMOPHYSICAL PROPERTIES OF MATERIALS}

》 DOI 10.1051/epn:2008501

\author{
H.-J. Fecht ${ }^{1}$, R. Wunderlich ${ }^{1}$, L. Battezzati ${ }^{2}$, J. Etay ${ }^{3}$, E. Ricci ${ }^{4}$, S. Seetharaman ${ }^{5}$ and I. Egry ${ }^{6}$, \\ ${ }^{1}$ Ulm University, Germany, ${ }^{2}$ University Torino, Italy, ${ }^{3}$ CNRS Grenoble, France, \\ ${ }^{4}$ CNR Genova, Italy, ${ }^{5} \mathrm{KTH}$, Stockholm, Sweden, ${ }^{6}$ DLR, Cologne, Germany
}

E or a wide range of new products in the industrial production chain, solidification processing of metallic alloys from the melt is a step of uppermost importance. Such advanced products are usually complex and made up of many components. Examples are:

- turbine blades for land-based power plants and for jet engines sustaining high temperatures and high stress levels,

- low-emission energy-effective engines for cars and aerospace, - so-called supermetals (amorphous metal alloys) such as thin sheets for electronic components with ultimate strength to weight ratio,

- high-performance magnets,

- biocompatible medical implants such as hip replacements,

- new semiconductors for solar cell applications,

- fine metallic powders to catalyse chemical reactions,

- new low-weight and high-strength materials for space exploration and future space vehicles.

Accordingly, to produce materials that meet ever-higher specific requirements, in particular to save energy by reducing weight or for use at high temperature, the solidification processing of structural materials has to be controlled with ever-increasing precision. This aim is most efficiently achieved by means of predictive quantitative numerical simulations of grain-structure formation using sophisticated integrated software, to improve casting quality and reproducibility, yield strength, creep resistance and other properties (Figure 1).

\section{Current limitations}

The application of such numerical tools is generally hampered by the lack of reliable thermophysical property values of the relevant liquid metals and alloys, needed as input parameters for the computational models $[1,2]$. In this respect, there is a discrepancy between the ever increasing sophistication of the numerical models and computer power, and the availability or accuracy of the thermophysical property data for the liquid phase. The paucity of data is generally owed to the high chemical reactivity of most metallic alloys in the liquid phase. This holds in particular for high-temperature alloys such as $T i$ alloys and $N i$-based superalloys, but also for some lower-temperature light weight alloys such as the $M g$ and $L i$ alloys. These are of interest to the automotive and aerospace industries. The current space-related research projects were conceived to improve this situation by combining conventional thermophysical property measurements with advanced containerless processing techniques of hot melts on ground and under reduced gravity conditions.

\section{Levitation of hot metallic drops in space}

Levitation of molten metals and other materials has been developed to solve a series of practical issues [1]. First, high-temperature chemical reactivity between liquid and container is often rather devastating (e.g. silicon in contact with graphite). The resulting sample contamination then drastically falsifies the measurements of thermophysical properties. Second, heterogeneous nucleation of solid phases easily occurs at the container wall. This makes deep undercooling and growth of metastable

$\nabla$ FIG. 1: MAGMASOFT ${ }^{\oplus}$ numerical simulation of the surface temperature distribution over a car engine block immediately after mould filling 

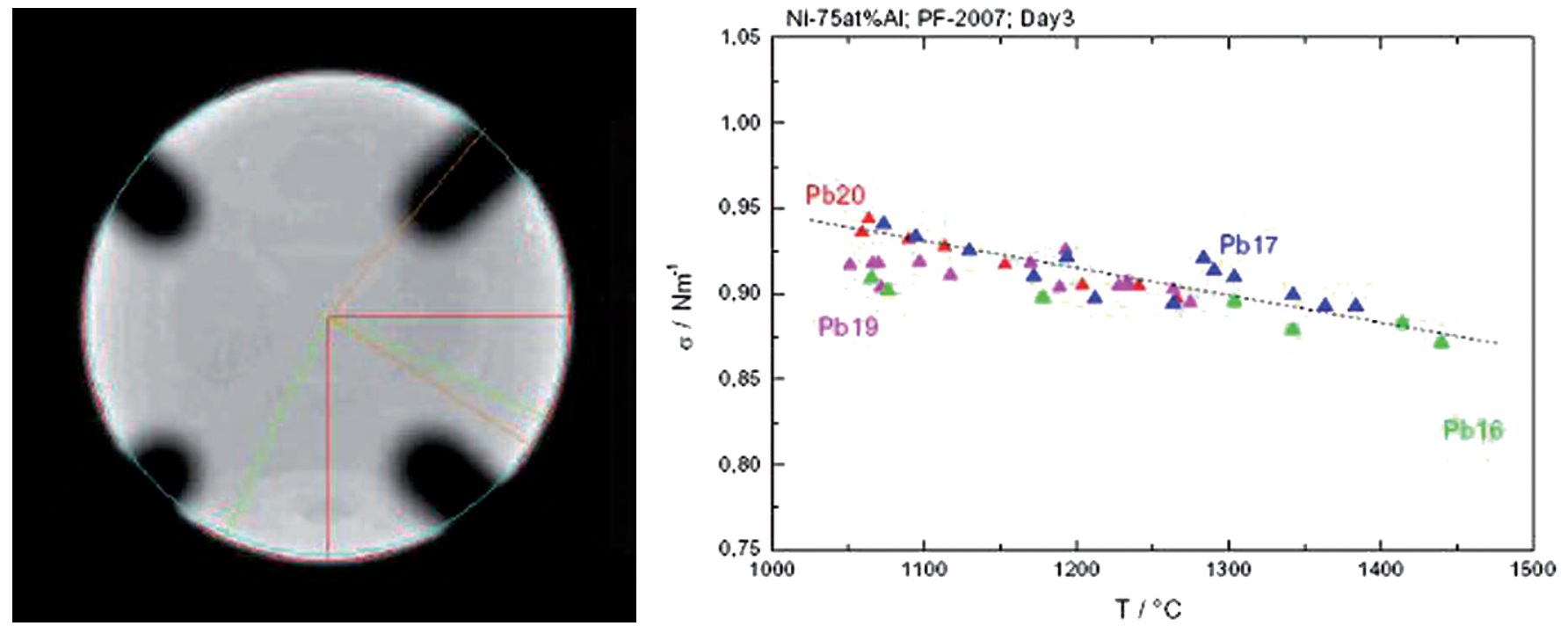

$\Delta$ FIG. 2: Left panel: Spherical droplet of a molten Ni-75\%Al sample (8 $\mathrm{mm}$ diameter) obtained in a parabolic flight. Video images allow measurements of the surface tension and viscosity as a function of temperature. Right panel: surface tension between 1050 and $1450{ }^{\circ} \mathrm{C}$ (the symbol Pb denotes the number of parabola performed during a single flight day)

phases unattainable. Conversely, without a container undercooling levels of several hundreds ${ }^{\circ} \mathrm{C}$ below the melting point can be achieved.

A reduced gravity environment adds further key merits to containerless levitation processing [1], namely:

- Achievement of perfectly spherical drop shape

- Levitation forces required to hold the samples are much lower than at 1-g conditions, resulting in improved temperature control (within $0.1 \mathrm{~K}$ ) and sample stability

- Improved control of sample environment (excellent vacuum conditions)

Furthermore, segregation and sedimentation effects become negligible, such that uniform mixing is achieved. Finally, the samples that can be levitated are much larger than on earth. Such experiments are, however, not exactly straightforward in microgravity where residual gravity and capillary forces at fluid surfaces are still at work. For example, everyone has seen on TV astronauts playing to catch large, freely floating drops of water. Nevertheless, stable sample positioning, which must be ensured to enable laser heating or pyrometry measurements, is possible with higher precision than on earth. In addition, it requires weaker electromagnetic fields. For more than twenty years, space agencies are thus developing levitators using electromagnetic forces, or alternatively acoustic waves or electrostatic forces. The ElectroMagnetic Levitator (EML) is taking advantage of well-known phenomena following from Maxwell's equations. High-frequency alternating fields are generated by passing a current through an assembly of coils shaped for positioning and heating. The applied electromagnetic field induces eddy currents in the metallic sample placed between the coils. This will heat, and may even melt, the sample, due to the Joule effect. The coupling of these currents with the applied electromagnetic field is used to impose a lifting force on the sample, which is concomitantly undergoing fluid flow driven by the electromagnetic forces when molten.

\section{Parabolic flight measurements}

Parabolic aircraft flights provide about twenty seconds of reduced gravity. This duration is sufficient for melting, heating into the stable, uniform liquid, and cooling to solidification of most metallic alloys of interest. It is thus well suited for the measurement of the surface tension and the viscosity by the oscillating drop method. In this method, surface oscillations are excited by a pulse of the heating field, and the surface tension and viscosity are obtained from the oscillation frequency and damping time constant of the oscillations, respectively. The process is implemented in an inert gas atmosphere that is circulated in order to enhance sample cooling and assure solidification at the end of the microgravity phase. This results in cooling rates between $30-50 \mathrm{Ks}^{-1}$.

Using the Electromagnetic Levitator, surface oscillations of the liquid hot drop with diameter of $8 \mathrm{~mm}$ (Fig. 2a) can be for instance introduced by an electro-magnetic pulse and the results analyzed by a high-speed high-resolution video camera $[5,6,7]$. Among the results, Figure $2 \mathrm{~b}$ shows the surface tension as a function of temperature in the range $1050-1450{ }^{\circ} \mathrm{C}$ of liquid $\mathrm{Ni}-75$ at $\% A l$ processed under low gravity in 4 parabolas with 10 seconds of processing time each.

\section{Critical technologies}

Therefore, one of the key ISS facilities under development is the ESA/DLR ElectroMagnetic Levitation (EML) device. This advanced EML will permit containerless melting and solidification of alloys and semiconductor samples, either in ultra-high vacuum or high-purity gaseous atmospheres over long time periods. Furthermore, the EML is equipped with highly advanced 
diagnostic tools which permit accurate measurements of thermophysical properties, as well as direct observation of the experiment during flight by high-speed high-resolution video analysis (see Figure 3).

\section{Summary and outlook}

Cast materials are common objects in everyday life (car and jet engines, metallic skeleton of buildings, dental or hip implants...). The latest developments have been achieved through solidification processing from the melt on earth and in space in order to improve casting facilities, and also by gas atomization and powder production for the catalytic activation of chemical reactions.

Ultimately, a reliable database of thermophysical properties of liquid metals and alloys over a wide range of temperatures will be established, including the following properties needed for numerical simulations and improvement of casting quality for new and advanced materials:

- density - using video observation techniques in the coil's axial and radial directions

- thermal expansion - also from video observation

- viscosity - using the oscillating drop technique

- surface tension - also by drop oscillation

- total hemispherical emissivity - using modulation calorimetry and measurement of external relaxation times

- specific heat - also by modulation calorimetry

- electrical conductivity - using inductive methods to measure the impedance of a pick-up coil surrounding the sample

- thermal conductivity - using the Wiedemann-Franz law that relates the electrical and thermal conductivity
- alloy melting range and fraction solid - using modulation calorimetry

In order to achieve these goals on a reliable basis sophisticated computer models have been recently favoured in Europe. These will, in all aspects of industrial materials processing, add to the long-term sustainability and technology leadership sought for in the worldwide competition. On this basis ESA together with national space agencies in Europe has set up a strong scientific programme to utilise space as a complementary crucial tool to ease and accelerate the development of new products through knowledge-based designs using the timely fundamental advances acquired from parabolic flights, sounding rockets and, in the near future, the International Space Station.

\section{References}

[1] D.J. Jarvis and O. Minster, Metallurgy in Space, Materials Science Forum 508 (2006), 1

[2] B. Billia and H.-J. Fecht, G. Seibert et al., A world without gravity (ESA SP-1251, ESA, Noordwijk, 2001), 186

[3] D. Herlach et al., Int. Mat. Rev. 38, (1993), 273

[4] T. Hibiya, I. Egry eds., Special Issue of Measurement Science and Technology 16-2, (2005)

[5] R. Aune et al. Superalloys 718, 625, 706 and Derivatives 2005, ed. E.A. Loria, TMS 2005, 467.

[6] K. Higuchi, H.-J. Fecht and R. K. Wunderlich, Advanced Engineering Materials 9 (2006), 349.

[7] R. K. Wunderlich et al. Solidification Processing 2007, ed. H. Jones, publ. Department of Engineering Materials, The University of Sheffield GB, 2007, 383.

V FIG. 3: Schematic diagrams of the Materials Science Laboratory, MSL (Left panel) and of the ElectroMagnetic Levitator, EML (right panel) both of which will be accommodated onboard the International Space Station.

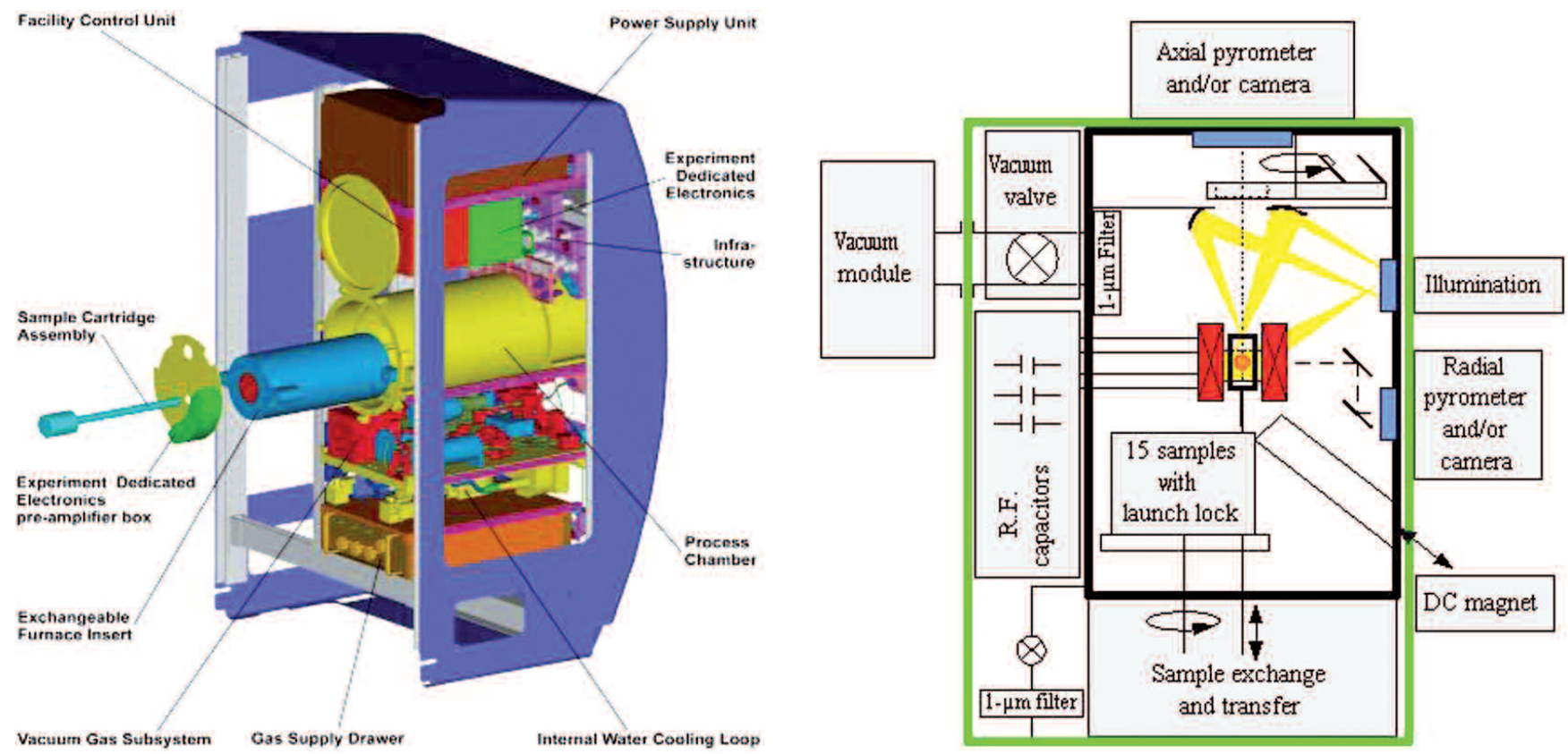

